\title{
SOME HIGH-VELOCITY SOURCES OF 1612 MHz OH EMISSION
}

\author{
F. J. KERR and P. F. BOWERS
}

University of Maryland, College Park, Md., U.S.A.

\begin{abstract}
A pilot survey was made to study the feasibility of using radio observations of $\mathrm{OH} / \mathrm{IR}$ stars to investigate the galactic distribution and kinematics of these presumably intermediate-population objects. Seven new sources were found, four near the center, two near $l=30^{\circ}$, and one at $l=128^{\circ}$.

Most identified OH/IR stars are believed to be long-period oxygen-rich Mira variables. Winnberg et al. (1973) have recently surveyed the galactic plane from $l \simeq 20^{\circ}$ to $50^{\circ}$ and have found about 30 new $1612 \mathrm{MHz} \mathrm{OH}$ sources. The lack of optical or infrared counterparts to the sources indicates that some of the sources may be quite distant since many $1612 \mathrm{MHz} \mathrm{OH}$ sources are also bright infrared objects and most of these sources have the microwave characteristics of OH/IR stars.

The velocities range up to $+140 \mathrm{~km} \mathrm{~s}^{-1}$, and in several cases the velocities are higher than would be expected for $\mathrm{H}_{\mathrm{I}}$ in the same direction, implying perhaps that these sources represent a population with a higher velocity dispersion than would be characteristic of Population I objects. It seems possible then to use radio observations of the $\mathrm{OH} / \mathrm{IR}$ stars to study the galactic distribution and kinematics of a disk popula-
\end{abstract}

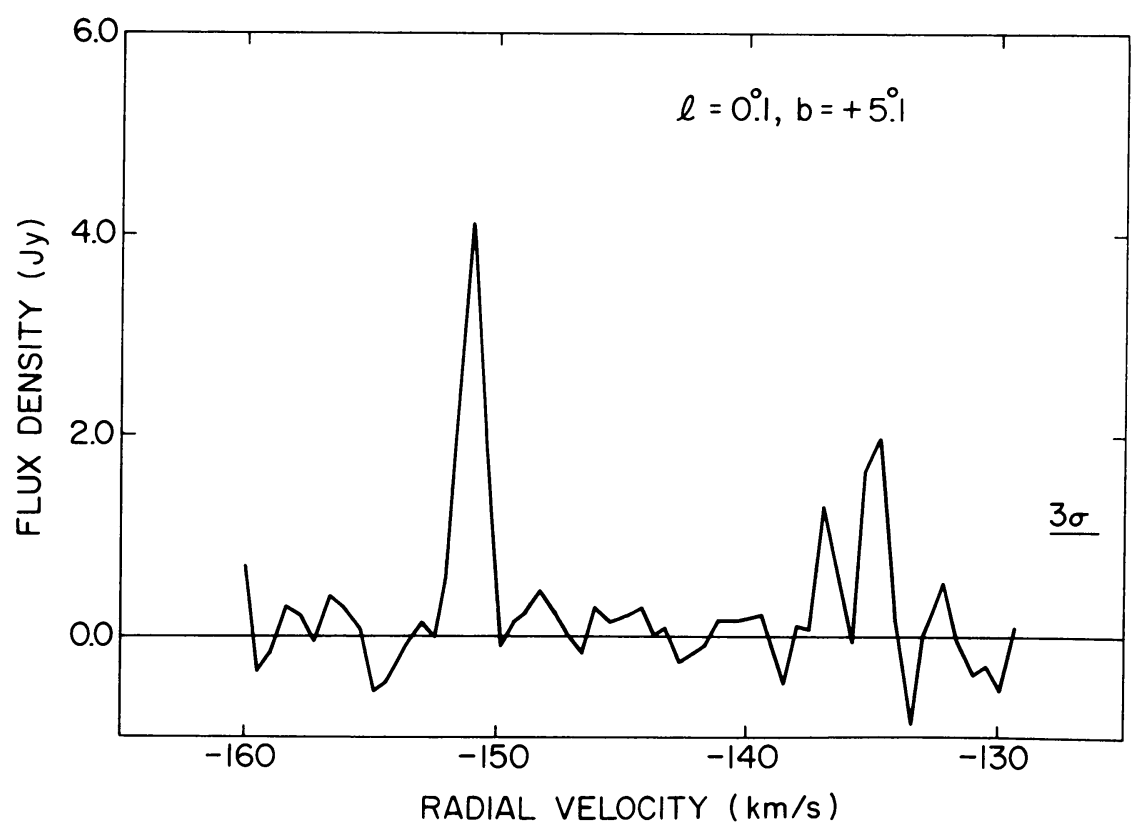

Figs. 1a-b. A $1612 \mathrm{MHz} \mathrm{OH}$ source in the general direction of the galactic center and (b) a $1612 \mathrm{MHz}$ source toward the outer part of the Galaxy. The galactic coordinates of each source are indicated. The velocity resolution is $0.67 \mathrm{~km} \mathrm{~s}^{-1}$, and the polarization angle was $0^{\circ}$. The radial velocities are with respect to the LSR. 


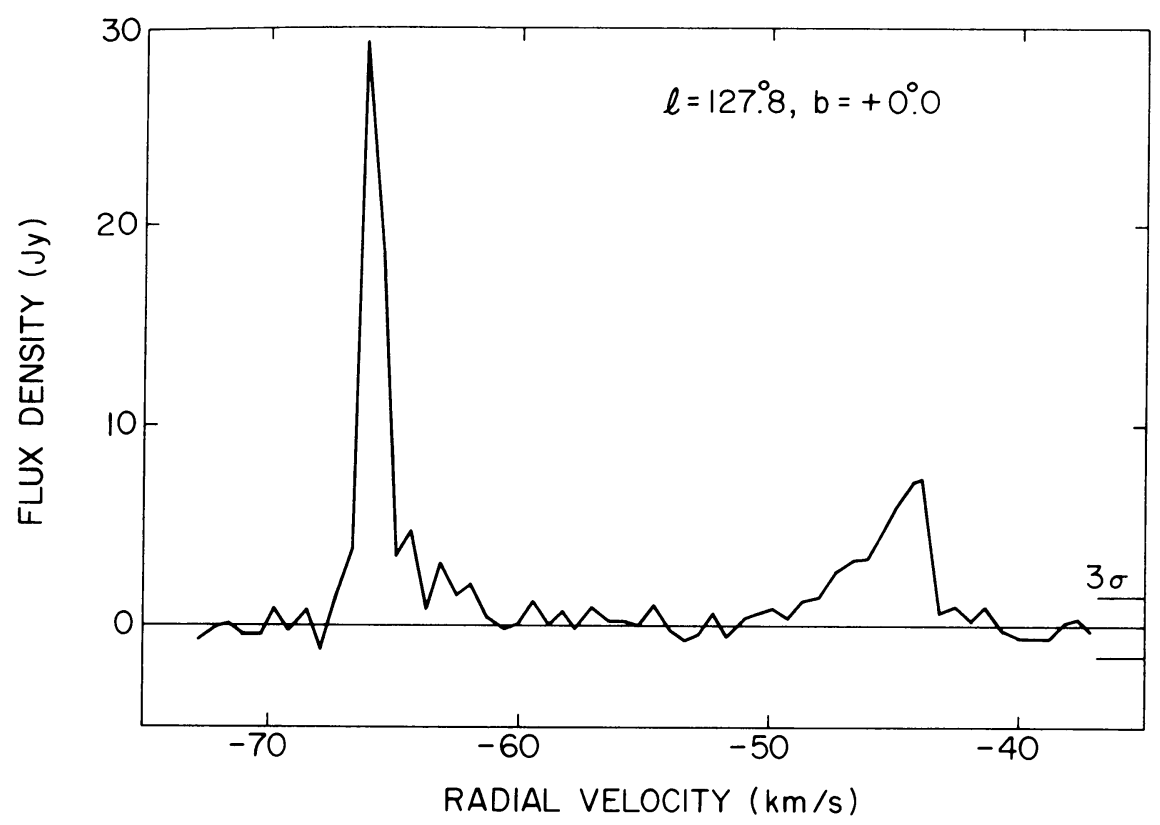

Fig. $1 b$.

tion. A pilot program has been completed to investigate the feasibility of such a project, using the $140-\mathrm{ft}(43-\mathrm{m})$ radio telescope at the National Radio Astronomy Observatory* in Green Bank, West Virginia.

Observations were obtained at about 150 positions. Over half of these were in directions in the vicinity of the galactic center, where an older population might be expected to be concentrated. Positions near $l=30^{\circ}$ and $l=130^{\circ}$ were also observed. Seven new sources were detected at $1612 \mathrm{MHz}$. Most of the sources have double peaks, as with the known OH/IR stars, and may be at large distances since there are no obvious optical or infrared counterparts.

Figure 1a shows the profile for a source which is especially interesting because this high velocity observed near zero longitude must be entirely due to a noncircular component of motion. Figure $1 \mathrm{~b}$ shows the profile of a strong source in a direction toward the outer part of the Galaxy, where little $1612 \mathrm{MHz}$ work has been done so far.

We are planning a larger survey to look for the stronger sources of this class. With respect to galactic structure, radio astronomy has hitherto been concerned primarily with Population I objects. The bright microwave emission from these sources may provide a new method for studying the distribution and kinematics of an older population.

In closing, we want to report briefly that a number of RV Tauri stars, some of which have large infrared color excesses, have been observed primarily at $1612 \mathrm{MHz}$ to a sensitivity of $\sim 1 \mathrm{Jy}$ (Bowers and Cornett, 1974). No OH emission was detected.

* NRAO is operated by Associated Universities, Inc., under contract with the National Science Foundation. 


\title{
References
}

Bowers, P. F. and Cornett, R. H.: 1974, Astrophys. Letters, in press.

Winnberg, A., Goss, W. M., Höglund, B., and Johansson, L. E. B. : 1973, Astrophys. Letters 13, 125.

\author{
F. J. Kerr \\ P. F. Bowers \\ Astronomy Program, University of Maryland, \\ College Park, Md. 20742, U.S.A.
}

\section{DISCUSSION}

Habing: You said that this is a pilot program, so you are going to extend this to the whole sky?

Kerr: Yes, we're extending it over the whole sky at low sensitivity, just looking for the strongest objects.

Caswell: I think that for the unidentified sources the major concern is whether the velocities are due to galactic rotation and give good kinematic distances. I believe the characteristic velocity of the objects is better represented by the mean velocity of the two $\mathrm{OH}$ peaks than by the red-shifted one, as is conventionally believed at present. We have six objects below the galactic center quite clearly well above the maximum velocity allowed as shown by the $\mathrm{HI}$ velocity distribution in this area which is very similar, symmetric about the galactic center. There is just nothing above the galactic center corresponding to these. However, there is a considerable increase in symmetry if you take the mean of the two $\mathrm{OH}$ peaks as the characteristic velocity of the objects.

Kerr: It's true that galactic rotation is certainly the most important component, and it can be argued which feature should be taken. If we take the extreme peak, that goes a long way outside the Schmidt curve. But I would like to point out that on the southern side the hydrogen distribution is somewhat different from the northern side in velocity. That's what the asymmetry is.

Palmer: How does the distribution of the newly discovered sources near the galactic center compare with that of the high velocity gas in the direction of the galactic center?

Kerr: There are not enough known yet for good statistics. However, some of the sources are high enough in latitude to be outside the normal gas layer, if they are at galactic center distances.

Robinson: Is it true to say that none of the Class $\mathrm{C}$ unidentified sources have been found in latitudes more than $3^{\circ}$ and they are all very close to the plane?

Caswell: That's true as far as I recollect, but there is a significant selection effect. The large number of search positions that has been done at high latitudes gives some indication that the unidentified sources are quite closely confined to the plane.

Kerr: Practically all searching has been done pretty close to the plane.

Caswell: The searching at the positions of long-period variable stars gives a mean galactic latitude of something like 6 . 